\title{
Improvement of Traceability Processes in the Farmed Fish Supply Chain
}

\author{
Alfredo Parreño Marchante ${ }^{1}$, Alejandro Alvarez Melcon'1, \\ Mira Trebar ${ }^{2}$, Andrej Grah², and Piero Filippin ${ }^{3}$ \\ ${ }^{1}$ Universidad Politecnica de Cartagena, Campus Muralla del Mar-Antigones, \\ Cartagena, Spain \\ ${ }^{2}$ University of Ljubljana, Faculty of Computer and Information Science, Tržaška cesta 25, \\ Ljubljana, Slovenia \\ ${ }^{3}$ University of Wolverhampton, School of Technology, Wulfruna Street, \\ Wolverhampton, Great Britain \\ alfredo.parreno@gmail.com, alejandro.alvarez@upct.es
}

\begin{abstract}
In the project "RFID from Farm to Fork" an implementation of RFID technologies are used along the food supply chain to be deployed in SMEs: from the farm to the consumer. As part of the project, two pilot deployments are being undertaken in the farmed fish business. The purpose of this paper is to show how the business process of the farmed fish supply chain can benefit from a novel system architecture that uses Radiofrequency Identification (RFID) and Wireless Sensor Networks (WSN) to improve the processes of fish traceability. In order to show the technological evaluation, both a definition of each company's business processes and how to upgrade them to the new technologies are presented.
\end{abstract}

Keywords: Traceability, farmed fish, supply chain, aquaculture, RFID, WSN.

\section{Introduction}

The production and distribution of food is becoming one of the most important issues in many countries all over the world [1]. The globalisation of this sector is creating concerns about the quality and the origin of the food products which is opening a place to collect as much information as possible from the whole supply chain.

In the past, several European projects were launched to explore the feasibility of RFID in supply chain. Among them, the eTrace initiative is to focus on the use of RFID technology to gain visibility in a variety of food supply chains throughout Europe. Another European project called TraSer [2] researched the linkage of the automatically retrievable identity of products to identity-specific tracking and tracing, and related web services. The output was an open-source TraSer community which, through its web services, assists the targeted group of users (mainly SMEs) to improve their efficiency in changing environments, such as supply chains, service 
operations and project delivery networks. This concept of traceability of product through web services is related to the project presented in this paper.

The paper presents two different pilot deployments of RFID and WSN technologies in farmed fish companies within the scope of the project RFID-F2F "RFID from Farm to Fork" [3]. The main objective is to define an applicable system which could perform the complete traceability by recording data at each stage. The pilots are deployed in two aquaculture companies in Slovenia and Spain and they are being undertaken by the University of Ljubljana and the Universidad Politécnica de Cartagena with the technical support of the University of Wolverhampton.

Firstly, the paper presents the supply chain model of each company and its business process. Afterwards, the RFID and WSN systems needed to automatize the collection of information along the supply chain are described. Finally, the novel system architecture proposed to integrate traceability information with WSN data through web-services is presented.

\section{Business Processes in Farmed Fish Supply Chain}

The basic objectives of each company are to capture data of a product moving upstream from a supplier to customer and to query data downstream to gain a shared view within the relevant business processes [4]. The supply chain model in the farmed fish industry has special characteristics due to the type of product that is being processed. The high requirements on the traceability process are due to short shelf life of the product. As a consequence, the use of cold chain throughout the entire supply chain must be maintained. The lead time from when the fish is caught to when it is shipped to the customer should be no more than 24 hours. All these requirements in the supply chain mean that any improvement in reducing the processing time of the fish increases product quality and, thus, the competitiveness of the company. Therefore, it is necessary for the producers to monitor and collect the information along the entire supply chain which includes the following stages: hatchery of young fish; on-growing farm; processing; warehousing; transportation and logistics; and retail.

Two pilots were carried out in Spain and Slovenia. The Spanish pilot was deployed in the company Culmárex which is an aquaculture enterprise that offers sea bream and sea bass in different sizes. The business processes that are relevant to the project and that will be improved with the introduction of WSN and RFID technology comprise two different locations, the on-growing farm and the processing plant. In the case of Slovenia, a small fish company Fonda.si in northern part of Adriatic Sea offers sea bass. The supply chain of the company comprises the on-growing farm, the processing plant, the logistics including warehousing of fish in cold store with distribution to retailers and also the sale at the fish market. 


\section{Traceability Solution}

In the pilots, RFID technology is used in all stages, starting from fish farms to the delivery to market. The main objective is to define an applicable system which could perform the complete traceability by recording data at each stage. Traceability and cold chain data are stored in a global database system accessible by each partner. Thus, the goal is to show that all parties, including consumers and producers would take advantage of such systems.

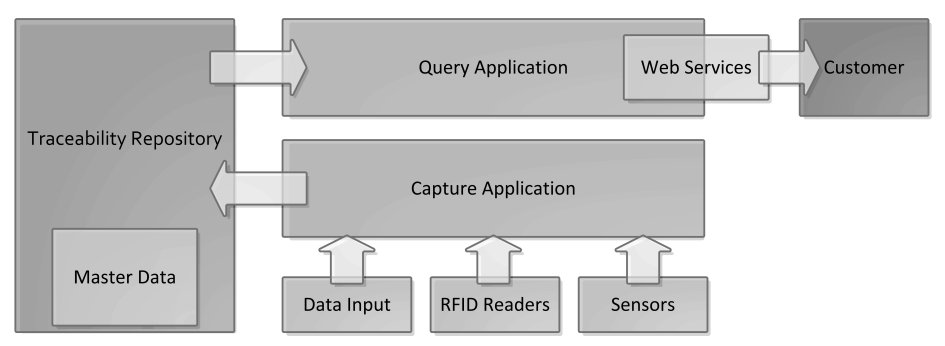

Fig. 1. RFID - Traceability system architecture.

In Fig 1 is shown the new traceability system architecture with four main components. The first one consists of RFID and sensor devices with other data inputs. In the second one capture and query applications are defined to store and retrieve the data from the repository. The third one is the traceability repository defined according to EPCIS standard which is used to store the relevant traceability data generated during the business process of the company [5]. The last component corresponds to web-services provided to create a service that presents all the information collected during the business process of a particular company to the relevant end-users, usually customers.

\subsection{Culmárex - Pilot Implementation}

The pilot implementation will comprise two different sites. The first one is the seaplatform where the on-growing and commercial cages are placed. The second one is the processing plant where the fish is packed in boxes for selling and stored in cold stores if necessary. The scope of the RFID pilot begins with the arrival of the juveniles to the on-growing farm and ends with the shipping of fish boxes to clients.

At the on-growing farm an RFID handheld reader is used. It allows storing information in a database at each step of the farm supply chain. Every cage is tagged with an RFID tag. A sensor logger is included in each tank to monitor the fish temperature in the transport from the farm to the processing plant.

At the processing and packing plant, RFID handheld and fixed readers are used. The implementation starts when tanks are received and their EPC's are read by an operator with an RFID handheld reader that uses an application form to store the data 
from the receiving process. Afterwards, tanks are identified by a gate with an RFID portal that reads every EPC of the tank tags as they enter through the gate. At the same time, the tank temperature logger is read and data are stored in a repository. When the fish enters the processing plant, it is automatically processed by a machine that sorts the fish by weight and the operators fill the boxes of fish and label them. The machine has printers for box labeling that admit the installation of an RFID module over them; therefore, every box of fish is tagged with an RFID tag. The memory of the tags is encoded with an EPC number, while the paper label is printed with the same information and barcodes used so far. Thereupon, boxes are moved to the conveyor belt where an RFID portal reads the tag to store the box EPC code in the repository, changing the state of the box in the system to ready for sale. For the shipping process, another RFID portal with a computer will be used. The software running on the computer allows inserting orders and checking them with the goods loaded on trucks.

Regarding cold chain requirements, temperature and humidity sensors are installed to control the environment of the processing, packing and cold store rooms.

\subsection{Fonda.si - Pilot Implementation}

The proposed RFID pilot of sea bass collects data from four stages of the supply chain: (i) Farm; (ii) Processing; (iii) Logistics; and (iv) Retail.

At the farm, the following actions are maintained during the process of breading sea bass: receiving juveniles at the farm, movement between cages, food and medicament information, and other inspections of cages (number of dead fish, nets replacement). On the harvesting days, the information of transporting sea bass in containers is included in the database. All these operations are implemented as applications on the RFID handheld reader. Cages on the farm are identified with RFID tags and scanned during each action in order to insert the information.

The processing consists of collecting customer orders, and processing-packaging fish for transport. The traceability data in the proposed RFID solution are collected during both processes. Each expanded polystyrene box of fish is attached with an RFID label with printed information as QR code where IDs will match.

All packed boxes are transported from the processing room to the cold store, stored there overnight and delivered to the customers (retail, restaurants, supermarkets and private customers) on the next day. An RFID portal (fixed RFID reader with two antennas) is used at the cold store door and connected to a computer running the application to control the shipping and receiving logistics processes.

Tracking of sea bass in the pilot solution ends with the delivery of boxes to the fish market where the application on an RFID handheld reader is used to read EPCs of boxes and temperatures measured with RFID data loggers during the logistics. 


\subsection{Customer Information}

As part of the system, it has been deemed meaningful and valuable to expose some of the collected information to the customer, with particular regard to product origin and conditioning. Doing so has been proven in other sectors to both raise the customer confidence on the companies involved and at the same time to increase the perceived value of the products.

To make it possible to link a particular product with the traceability backend, items have to carry, up to the retail point, a machine readable ID which identifies the smallest logistic/traceability unit the system can supply information about. This ID can in general be carried in the form of a barcode (linear or 2D-dimensional), an RFID tag (UHF or NFC) or a mixture of the above where and when convenient. For our pilots, the choice for the primary customer-facing identifier has fallen on QR Code, which allows the desired information to be conveyed to any QR Code-capable smartphone, removing the need for the installation of specialist equipment at the point of sale (fish market/retail store/restaurant).

Upon scanning, the ID is passed by the smartphone to the online traceability system, which collates all the information from the entities that have been involved in handling the product (fishing boats, processing plant, logistics etc.). This information mostly comprises of times and dates, names of processing steps and states, locations and sensor data (temperatures). The information is then transformed into a visual representation in form of a web page - images, graphs, maps, diagrams and descriptions and presented to the customer along with a "traffic-light" coded signaling system which shows, at a glance, how close to the ideal range each piece of information is.

\section{Conclusion}

The project 'RFID from Farm to Fork' proposed the aforementioned pilot implementations in the farmed fish supply chain as a means to highlight the use of RFID and sensor technology in conjunction with a global EPC-compliant structure to collect, store and display the data from the farm to the end customer. The proposed RFID solution, composed of hardware and software modules, is currently in the implementation phase after having been tested in a laboratory setup. In the next phase, the goal is to transfer the RFID system framework to a full integration with the enterprise information systems. The smaller pilot in Slovenia is already deployed in the company where more tests are accomplished to measure the performance of the system, test and improve applications and evaluate the implementation. The adopted solution offers an example of a flexible, scalable and interoperable system that can be easily transferred to any business processing of farmed fish.

Acknowledgments. This work has been supported by European Union, under the project name "RFID from Farm to Fork", grant agreement number 250444. 


\section{References}

1. Ward V., Consumer interest in information cues denoting quality, traceability and origin: An application of ordered probit models to beef labels (2005)

2. EU FP6 funded project "TraSer" ICT for Networked Businesses Programme. Available: http://www.traser-project.eu/ (2012)

3. 5. EU Project "F2F-RFID from Farm to Fork". ICT PSP Programme, 2010. Available: http://www.rfid-f2f.eu (2012)

4. Swedberg, C.: "Scandinavian Group Finds That Tracked Cod Sells Better", RFID Journal. Available: http://www.rfidjournal.com/article/view /7699 (2010)

5. Thakur, M., Ringsberg, H.: "Impacts of using the EPCIS standard in two fish supply chain”, Food Integrity and Traceability Conference, Belfast, (2011) 21-24 